\title{
Research with High-Power Short-Wavelength Lasers
}

\author{
J. F. Holzrichter \\ E. M. Campbell \\ J. D. Lind 1 \\ E. Storm
}

Manuscript Date: March 5, 1985

\begin{abstract}
DISCLAIMER

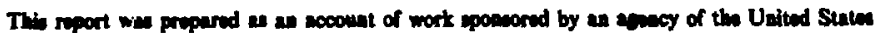

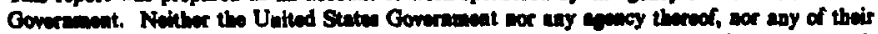

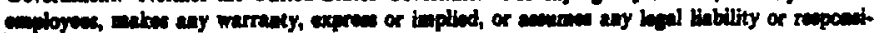

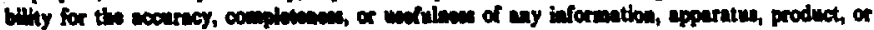

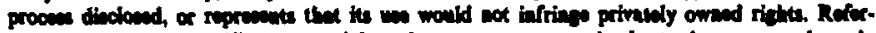

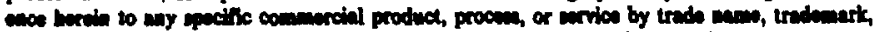

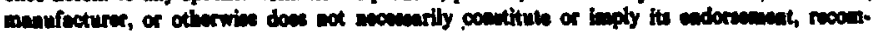

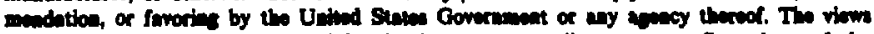

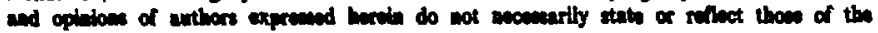

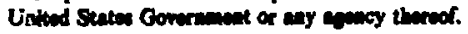

\section{LAWRENCE LIVERMORE NATIONAL LABORATORY University of California - Livermore, California • 94550}




\section{Foneword}

Access to states of matter seen only in stellar media or thermonuclear explosions, and the lure of virtually inexhaustible controlled fusion power, have motivated the construction of high-power lasers during the last decade. A sequence of ever more powerful machines has evolved at the Lawrence Livermore National Laboratory (LLNL), each taking advantage of information gathered with its predecessor and each providing more target-physics information. The largest and most recent laser of this series is a 10-beam, 50 - to $100-\mathrm{kJ}$ machine called Nova, now beginning to irradiate its first targets.

From the review paper by J. H. Nuckolls, L. L. Wood, A. R. Thieasen, and G. B. Zimmerman in 1972 to the present day, it has been clear that more than $1 \mathrm{MJ}$ would be needed to demonstrate high gain in an inertially confined fusion plasma. Controversy over where ignition and low gain would occur raged throughout the 1970s; the answer is still uncertain, because minimum ignition boundaries are very difficult to determine. Like its smaller antecedents (Novette, Shiva, and Argus), Nova is a research machine, dedicated to studying the physics of plasmas at high temperatures, pressures, and densities to accurately predict the gainvs-energy relation for ICF research and to obtain physics information for weapons applications.

The Nova laser, the result of an ambitious effort that has taken six years to complete, relied on the extension of many new technologies. The first-delivered Nova components were combined with salvaged parts from earlier laser systems into a two-arm tesi bed laser called Novette. Novette was used to obtain early information on Nova laser chain performance and on the performance of targets at short wavelengths. The studies described in this report were carried out with Novette.

The Novette team, guided by K. R. Manes, C. R. Speck, and G. J. Suski, called on the resources of the Nova project, led by R. O. Godwin and W. W. Simmons. Nova's lead engineers, E. S. Bliss, F. W. Holloway, F. Reinecker Jr., J. R. Severyn, M. A. Summers, E. P. Wallerstein, and K. Witham and their groups focused the efforts of a sizable segment of U.S. high-technology industry on the rapid construction of this laser. In the thirteen months it took to complete Novette, ending in early January 1983, most of the innovations essential to Nova's subsequent operation were realized in integrated, functic ing hardware in the Novette experiment.

Intensive target experinentation began with Novette in late January 1983 under the direction of L. W. Coleman. D. L. Matthew: led an early effort to obtain X-ray laser data with instruments specially designed by R. L. Kauffman and C. Wang. This experimentation was quickly followed by laser-plasma interaction studies by R. P. Drake, R. E. Turner, W. L. Kruer, K. G. Fstabrook, B. F. Lasinski, and R. L. Kauffman. Latt in 1983, studies of indirectly driven inertial-confinement fusion by F. Ze, L. J. Suter, and T. P. Bernat $2 \mathrm{~d}$ to our best results on highly compressed ICF tamets. Early in 1984, laser-plasma interaction and hohlraumscaling experiments were carried out at the fourthharmonic frequency of the Novette laser. The final experiment was a successful second $x$-ray laser investigation conducted by $D$. L. Matthews, M. D. Rosen, and P. L. Hagelstein, with speciai diagnostic support from N. M. Ceglio, M. J. Eckart, and $\mathrm{H}$. Medecki. The many very elaborate targets were provided by the Target Fabrication Group, led by I. F. Stowers and C. W. Hatcher.

Each of these campaigns was supported and made possible by the Novette Operations Group, which included O. C. Harr, D. G. Gritton, and J. S. Hildum and a number of employees of Bendix Field Operations, Inc.

The authors, who were responsible for the various elements discussed in this report, are indebted to their colleagues mentioned above and to many more, unnamed here, who supported this effort. They wish to thank J. L. Emmett, Associate Director for Lasers, for his continued support and technical contributions, and W. Krupke for his contributions to laser science. They also wish to thank LLNL's senior management-R. E. Batzel, A. C. Haussmann, J. H. Nuckolls, and R. D. Woodruff-and the Office of Inertial Fusion, Department of Energy, for their continued support. 


\section{Contents}

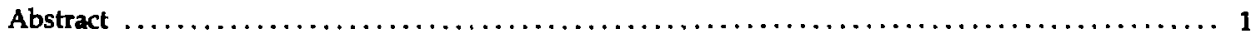

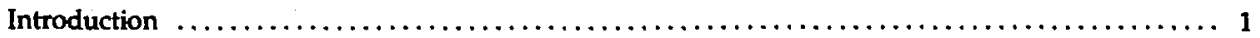

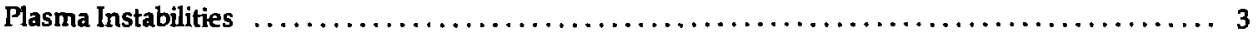

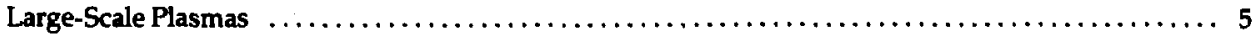

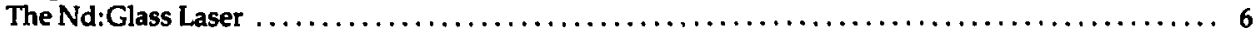

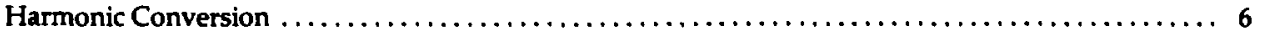

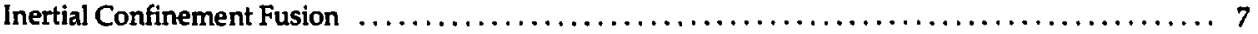

Compression $\ldots \ldots \ldots \ldots \ldots \ldots \ldots \ldots \ldots \ldots \ldots \ldots \ldots \ldots \ldots \ldots \ldots \ldots \ldots \ldots \ldots \ldots \ldots \ldots \ldots \ldots$

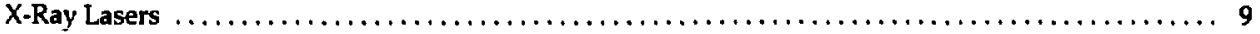

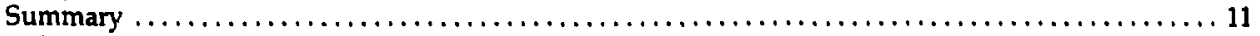

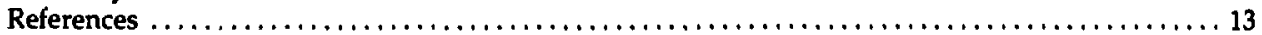




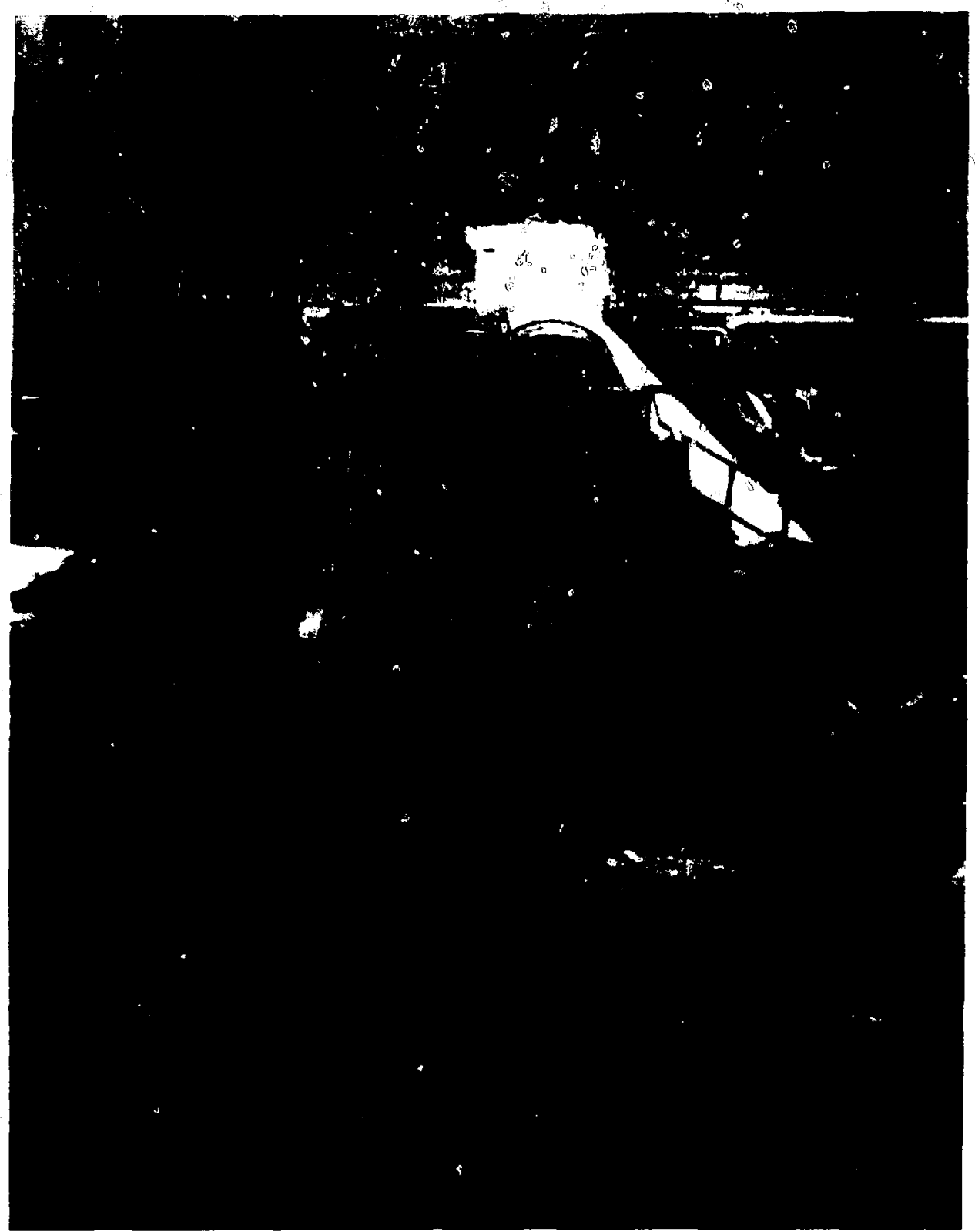

Frontiepiece. The two arms of the Novette laser, folded twice to fit into the available space, extended into the background. Novette delivered $9 \mathrm{~kJ}$, 1-ns laser pulses at $0.53 \mathrm{~m}$ to targets in the interaction chamber in the foreground. 


\title{
Research with High-Power Short-Wavelength Lasers
}

\begin{abstract}
Three important high-temperature, highdensity experiments were conducted recently using the 10-TW, short-wavelength Novette laser system at the Lawrence Livermore National Laboratory. These experiments demonstrated successful solutions to problems that arose during previous experiments with long wavelength lasers $(\lambda \geq \mid \mu \mathrm{m})$ in which inertial confinement fusion (ICF), $x$-ray laser, and other high-temperature physics concepts were being tested. The demonstrations were: (1) large-scale plasmas (typical dimensions of up to 1000 laser wavelengths) were produced in which potentially deleterious laserplasma instabilities were collisionally damped. This experiment and associated modeling indicates that the laser-plasma coupling in nigh-gain ICF target plasmas, which will have typical dimensions of $10^{4}$ wavelengths, will also be collisionally dominated. (2) Deuterium-tritium fuel was imploded to a density of $20 \mathrm{~g} / \mathrm{cm}^{3}$ and a pressure of $10^{10} \mathrm{~atm}$. This density is within a factor of 10 of the 1000 times liquid density required for the success of inertial confinement fusion, and the

compression conditions (preheat and pressure) are consistent with achieving efficient burn-up of a larger thermonuclear fuel mass when driven with a larger, short-wavelength laser. (3) A 700fold amplification of soft $x$ rays by stimulated emission at 206 and $209 \AA$ (62 eV) from $\mathrm{Se}^{+24}$ ions was observed in a laser-generated plasma. Isuelectronic scaling to $155 \AA(87 \mathrm{eV})$ in $\mathrm{Y}^{+29}$ was also demonstrated. These small, short-pulse $x$-ray sources are $10^{10}$ to $10^{11}$ times brighter than the most powerful $x$-ray generators and synchrotron sources available today. The plasma conditions for these experiments were made possible by advances in the technology of $\mathrm{Nd}$ :glass lasers and in techniques for efficiently generating their shortwavelength harmonics at $0.53,0.35$, and $0.26 \mu \mathrm{m}$, as well as improvements in diagnostic and computational modeling. We conclude that high-power lasers with output wavelengths between 0.53 and $0.26 \mu \mathrm{m}$ generate plasma conditions suitable for high-gain fusion, long-wavelength $x$-ray lasers, equation of state measurements, and other applications.
\end{abstract}

\section{Introduction}

The development of high-peak-power lasers in the mid 1960 s made it possible to create, in the laboratory, states of matter that had previously existed only under astrophysical conditions or in nuclear explosions. The anticipated ability to create such conditions on large scales $(-1 \mathrm{~cm})$ engendered a variety of technological proposals, including laser-driven thermonuclear implosions ${ }^{1-3}$ and $x$-ray lasers. ${ }^{4,5}$ Because high-quality laser light can be focused easily to dimensions less than $30 \mu \mathrm{m}$, early lasers (typically delivering $1 \mathrm{~J}$ of energy in a $10^{-9}-\mathrm{s}$ pulse) generated intensities of over $10^{14} \mathrm{~W} / \mathrm{cm}^{2}$. This intensity was sufficient to heat matter in the focal region to temperatures approaching $1 \mathrm{keV}\left(10^{7} \mathrm{~K}\right)$, which are required for many of the proposed applications. In these early experiments, much of the physics of interactions between high-intensity lasers and matter was examined. ${ }^{6}$ These experiments added impetus to the technological proposals, but they indicated also that there was nonclassical or anomalous behavior in the laser-plasma interaction ${ }^{7}$ The small size of the experimental plasmas $(-30 \mu \mathrm{m})$ precluded testing the key physical models.

By the early 1970s, Nd:glass lasers at wavelengths of $1.06 \mu \mathrm{m}$ were being used to conduct experiments with a few tens of joules in 0.1-ns pulses. This output gave $10^{17} \mathrm{~W}$ of power $(0.1 \mathrm{TW})$, which when focused to a spot size of about $100 \mu \mathrm{m}$ gave a peak intensity of up to $10^{15} \mathrm{~W} / \mathrm{cm}^{2}$. When the light from these systems was focused onto small glass microbailoons containing deuterium and tritium (DT) gas, fusion conditions were achieved. Neutrons generated by thermonuclear fusion were first reported by workers at KMS Fusion, ${ }^{8}$ and soon thereafter at LLNL $^{9}$ (where their origin was confirmed) and elsewhere. ${ }^{10}$

These experiments gave an early impetus to ICF research and to researchers planning to use lasers to create high-temperature, high-density states of matter for shock-wave, hydrodynamics, atomic-physics, and $x$-ray physics research. 
However, theoreticians noted ${ }^{11,12}$ that in the larger plasmas $(\sim 1 \mathrm{~cm})$ that were necessary for full-scale demonstrations of these technologies, very nonthermal conditions could occur if the laser wavelength - or the plasma - were too large. Laserplasma instabilities could grow large if insufficiently damped, and a very-high-energy electron population could develop. When laserplasma experiments were conducted with 100 - to 1000-J, 0.1- to 0.5-ns lasers, many targets showed energy partitioning into the suprathermalelectron population exceeding $50 \%$ of the absorbed energy, levels of backscattered laser radiation exceeding $40 \%$, and poor conversion of laser light into thermal $x$ rays. Early hopes for high fuel compression and efficient thermonuclear-neutron production from ICF targets using the 10-kJ Shiva laser $^{13}$ at $1.06 \mu \mathrm{m}$ and the Helios laser ${ }^{14}$ at $10.6 \mu \mathrm{m}$ could not be supported in light of these experimental observations. In addition, relatively straightforward physics measurements (e.g., shock-wave velocities and atomic-physics spectral studies) were complicated because of the presence of very energetic plasma electrons. In spite of the difficulties in using 1- $\mu \mathrm{m}$ light to develop good compression conditions, progress in understanding plasma scaling was made using $1-\mu \mathrm{m}$ and $10-\mu \mathrm{m}$ lasers. Our colleagues at LLNL demonstrated ${ }^{15}$ DT fuel compression in an ICF target that approached $20 \mathrm{~g} / \mathrm{cm}^{3}$, or 100 times liquid-DT density; but the target was complex, and the compression conditions were not suitable for efficient fusion. By using low-energy (1- to 100-J), short-wavelength laser light and continuing analysis, further progress in understanding the interplay and coupling between the laser light and the plasma was made. ${ }^{10}$ It became clear that larger, short-wavelength lasers $(\lambda<1 \mu \mathrm{m})$ were required for continued progress in this field.

With the present generation of high-power lasers $_{i}{ }^{16,17}$ we can produce energies in the range of 10 to $100 \mathrm{~kJ}$ at wavelengths $0.5 \mu \mathrm{m}$ or shorter and with pulse duration of about $10^{-9} \mathrm{~s}$. These lasers, in conjunction with sophisticated diagnostic and computational capabilities, have made it possible to address fusion and x-ray laser proposals at scales large enough to test many of their basic premises (see Fig. 1). Using the Novette laser (shown in the frontispiece) over the 1- to 10-kJ energy and 0.26 - to $0.53-\mu \mathrm{m}$ wavelength ranges, we produced collisionally stabilized thermal plasmas with interaction lengths of $10^{3}$ laser wavelengths. We used these plasmas to obtain compression conditions similar to those needed for efficient thermonuclear fuel burn, and we implcded DT fuel

\section{(a) ICF tergat}

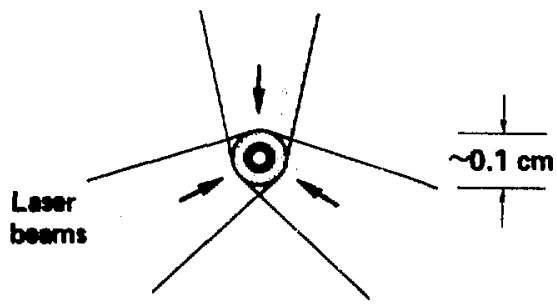

(b) $X$-ray leser tergat

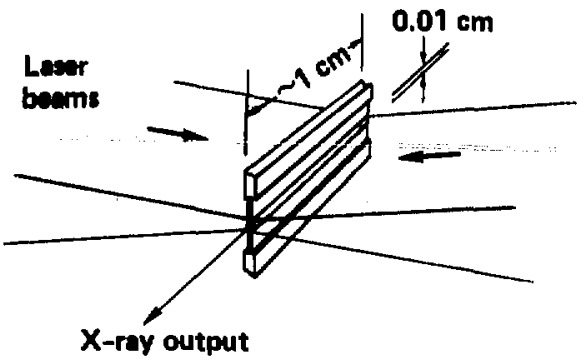

Fig. 1. (a) High-gain inertial confinement fusion targets start with solid-density DT fuel contained in an ablator. (b) Soft $x$-ray laser targets consisted of thin films of selenium or yttrium irradiated by line-focused laser beams. Dimensions in (a) and (b) are typical of current laser-target experiments.

capsules to densities exceeding $20 \mathrm{~g} / \mathrm{cm}^{3}$. By bringing the $0.53-\mu \mathrm{m}$ laser light to a line focus we produced conditions in which we measured 700 -fold amplification of soft $x$ rays at 206 and $209 \AA$ in 24 times ionized selenium targets.

In addition to these ICF and x-ray laser experiments, large lasers are being used to make measurements in a variety of other scientific disciplines. Interesting examples include the acceleration of metal foils (mass $10^{-6} \mathrm{~g}$ ) at $10^{16} \mathrm{~cm} / \mathrm{s}^{2}$ $\left(10^{13} \mathrm{~g}\right)$ to speeds of near $10^{7} \mathrm{~cm} / \mathrm{s}$, ${ }^{18}$ shock pressures of over $70-\mathrm{Mbar}^{19}$ and $\mathrm{x}$-ray sources ${ }^{20}$ with brightnesses of over $10^{14} \mathrm{~W} \cdot \mathrm{cm}^{-2} \mathrm{sr}^{-1}$ and with pulse durations from $10^{-10}$ to $10^{-8} \mathrm{~s} .^{21}$ As a result of these recent successes at LLNL, and previous work conducted at many laboratories around the world, ${ }^{10}$ we conclude that lasers with wavelengths of $0.5 \mu \mathrm{rn}$ or shorter are required for many applications of high-power lasers, such as ICF or x-ray lasers. 


\section{Plasma Instabilities}

The process by which laser light deposits energy in the target plasma determines the subsequent hydrodynamic motion, the atomic physics, and the energy transport. All these processes are strongly affected by the electron and ion energy distributions produced during the absorption process. An understanding of this interaction at the high intensities required for many applications has been difficult to obtain because numerous collective plasma processes (parametric instabilities and laser self-focusing) can be strongly excited. ${ }^{12,22}$ In the extremely nonuniform plasmas typical of ICF applications, it has been particularly difficult to predict the thresholds for the onset of these processes and their nonlinear saturation levels. A major objective of researchers in this field has been to understand these processes.

Laser energy couples to a plasma through a variety of processes that can be grouped generally into two categories, collisional and collective. In collisional processes the plasma electrons, oscillating in the laser electric field, are scattered by the much heavier ions, thus converting the coherent laser energy into random (thermal) energy. This process, called inverse bremsstrahlung absorption, can be extremely effective in absorbing laser light and in producing a thermal distribution of electrons. The characteristic energy of these electrons is 1 to $5 \mathrm{keV}$, depending on the laser wavelength and intensity and on the various dissipative mechanisms in the plasma. (radiation, hydrodynamic expansion, and thermal conduction). For the majority of applications, and for laser fusion in particular, inverse bremsstrahlung is the desired coupling mechanism.

In contrast, collective (or collisionless) absorption processes can lead to the generation of highly energetic electrons, with typical energies in the range of 10 to $100 \mathrm{keV}$. This type of coupling arises from the long-range nature of the Coulomb force, which allows plasmas to support both low- and high-frequency charged-particle oscillations without collisional interaction. These plasma waves can be driven to large amplitudes by the highintensity, rapidly varying electric field of the laser. The electric field associated with the highrequency plasma density fluctuations can effectively accelerate (heat) electrons, particularly those traveling near the phase velocity of the plasma waves. Reducing the laser intensity below $10^{14} \mathrm{~W} / \mathrm{cm}^{2}$ (and thus reducing the driving electric field of the laser) to avoid instability generation unfortunately reduces the pressure below the nominal 50 Mbar required to implode fusion capsules. The laser energy that can be delivered to the target before performance is affected by plasma instabilities depends on the laser wavelength, the size of the plasma, and the target average "atomic number" (average ionic charge) $\bar{Z}$.

The plasma instabilities can be characterized by tha various daughter waves into which the incident laser photons decay. ${ }^{23}$ The Raman, Brillouin, and $2 \omega_{p e}$ instabilities play a major role in laserplasma coupling. The Raman instability represents decay of the laser light into an electronplasma wave (or Langmuir wave) and a scattered photon; Brillouin scattering is decay into a scattered photon and an ion-acoustic wave; and the $2 \omega_{\text {pe }}$ instability is decay into two plasma waves. The electron plasma waves resulting from the Raman and $2 \omega_{\text {pe }}$ instabilities generally damp by accelerating electrons in the plasma. This collisionless, or Landau, damping can produce very energetic electrons because the phase velocity of the plasma waves is usually much greater than the electron thermal velocity. The traveling charge gradients caused by the electron plasma waves can generate longitudinal electric field stren'sths as great as $10^{9} \mathrm{~V} / \mathrm{cm}$, which accelerate slower electrons. The resulting suprathermal electrons can penetrate throughout the pellet, causing preheating that increases the entropy of the fuel and makes energy-efficient compression much more difficult. While this acceleration mechanism is extremely detrimental to the fusion application, it is being considered as a means of particle acceleration in high-energy physics research. ${ }^{24}$

Brillouin scattering, while not capable of producing suprathermal electrons, can also prevent effective laser-plasma coupling. It produces an ion-acoustic wave (the plasma analogue of a sound wave) that has a low phase velocity and that couples very well to the plasma ions. However, because of the low frequency of this wave compared with that of the incident laser wave $\left(\omega_{\mathrm{ia}} / \omega_{0}<10^{-3}\right)$, very little energy is carried by the ion-acoustic wave to be transferred to the plasma. The scattered Brillouin light wave carries away most of the energy, seriously reducing the absorption efficiency.

Short-wavelength laser light reduces the effects of these instabilities in several ways. First, the light interacts with the matter at higher densities, because the maximum electron density to which the laser can penetrate in the plasma is 
$n_{c}=10^{21} \lambda^{-2} \mathrm{~cm}^{-3}$,

where $\lambda$ is in micrometers. At this density, the electron plasma frequency is equal to the laser frequency, and laser-light propagation stops. However, because shiort-wavelength light propagates to a higher plasma density, where the electron-ion collision rates are higher, more light is absorbed along the propagation path via inverse bremsstrahlung.

Another consequence of the propagation to increased density is that the net growth rates of the unstable plasma waves are reduced because of increased electron-ion collision damping.

A further benefit of short-wavelength operation is that the transverse electron velocity, driven by the oscillating laser field, is reduced. This velocity is proportional to $\left(I \lambda^{2}\right)^{1 / 2}$, so that at constant intensity it drops as $\lambda$ is decreased. Since this is the forcing function for the plasma instabilities, the instability growth rate also drops.

A final consequence is that, with constant absorbed intensity, the higher-density plasma remains cooler, so that plasma-wave phase velocities are somewhat lower and the energy of the accelerated suprathermal electrons is less. This results in lower preheat for a given number of suprathermal electrons, since their penetration range scales as $E^{1.5-2.0}$. Figure 2 summarizes the effectiveness of laser-plasma coupling using short-wavelength lasers.

Kruer $^{25}$ has given a map of plasma instability thresholds that self-consistently includes the above arguments. The map is shown in Fig. 3. In this figure the instability thresholds for a gold plasma ( $\bar{Z}=30$ to 50 ) are plotted using the two variables that most often dominate the threshold alternatives, the plasma size relative to the laser wavelength and $I^{2}$, which determines the transverse electron velocity. Similar maps are available for plasmas with other $\bar{Z}$, in which the collision frequency (which is proportional to $\bar{Z}$ ) is different.

Figure 3 shows that plasmas formed under conditions far to the right of the instability thresholds have a disproportionate number of suprathermal electrons. Plasmas formed to the left of these lines have classical absorption and are largely thermal. On the left, the thresholds are determined by plasma collisionality, and they can become independent of plasma size and rise vertically (top left side of figure). Computer simulations and theory show that in this regime, when the electron-ion collision frequency is comparable to the instability growth rate, the instabilities are effectively damped and cannot become very efficient. High-gain targets must operate in this regime. Thresholds to the lower right in Fig. 3 are dominated by the relatively small laser-plasma interaction length ( $L / \lambda$ small). This limits the length in the plasma over which the laser and daughter waves resonantly interact and exchange energy. Thus plasma gradients, which limit the interaction length, provide another effective damping mechanism. Experiments conducted between 1979 and 1981, with 10- to 100-J lasers at 1.06, 0.53, and $0.35 \mu \mathrm{m}$, probed this region and demonstrated the efficiency of short-wavelength coupling to plasmas at small scales.

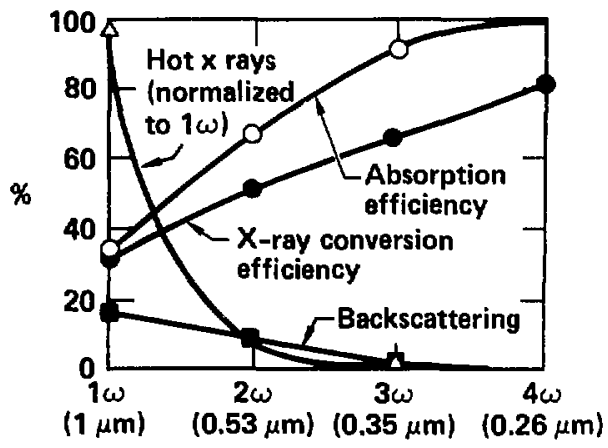

Fig. 2. Laser-to-plasma coupling to gold disks at intensities of $3 \times 10^{14} \mathrm{~W} / \mathrm{cm}^{2}$ (500-ps pulses) improves as the laser wavelength is decreased, and the signatures of plasma instabilities (energetic $x$-rays and scattered laser light) drop sharply. 
Fig. 3. Laser-plasma interaction regimes for collisional and collective absorption processes. Absorption occurs primarily by collisions for experimental conditions to the left of the lines, and more and more strongly by collective instabilities as experimental conditions move to the right. Data points represent experiments on gold plasmas with scalelengths of $L / \lambda \simeq 1000$. In the collisionally stabilized experiment, less than $0.001 \%$ scattered Raman light and no hot electrons were observed.

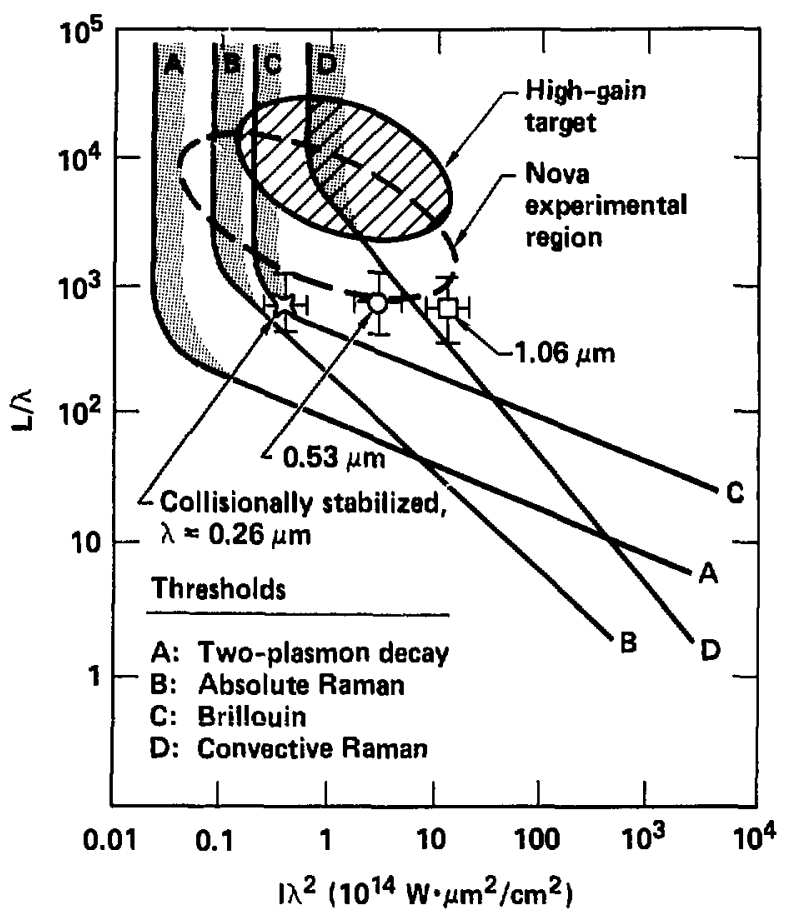

\section{Large-Scale Plasmas}

Recent experiments performed with the Novette laser at 0.53 and $0.26 \mu \mathrm{m}$ (and earlier on Shiva at $1.06 \mu \mathrm{m}$ ) demonstrate the sensitivity of the laser-plasma coupling to the above variables over plasma dimensions of $10^{3}$ laser wavelengths. ${ }^{26}$ Films of $\mathrm{CH} G \mu \mathrm{m}$ thick $(\bar{Z} \sim 3)$, and gold films $0.4 \mu \mathrm{m}$ thick $(\bar{Z}=30$ to 50$)$, were irradiated and expanded so the density dropped below the critical density, leading to long interaction lengths. In experiments in which plasma inhomogeneity and collisional thresholds were exceeded at 1.06 and $0.53 \mu \mathrm{m}$ on such targets (see Fig. 3), stimulated Raman scattering efficiencies as great as $3 \%$ to $10 \%$ of the laser energy were observed. Even using $0.26-\mu \mathrm{m}$ light and the $\mathrm{CH}$ target, efficient scattering was observed. However, in experiments with gold plasmas and $0.26-\mu \mathrm{m}$ light (again see Fig. 3 ) in which plasma instability thresholds due to inhomogeneity were exceeded but collisional damping was dominant, the Raman scattering efficiency was reduced to less than $0.001 \%$.
High-energy $x$ rays, the signature of hot electrons, were not measurable in these latter experiments. Because of their high absorption and low hot-electron density, these collisionally stabilized plasmas provide satisfactory operating conditions for ICF implosions.

The demonstration of collisional stabilization is extremely important, because high-gain targets driven with multi-megajoule lasers will have longscalelength $\left(L / \lambda=10^{4}\right)$ plasmas. Figure 3 indicates that the expected operating regime is somewhat above the instability thresholds. Numerical simulations and the experiments just described indicate that even though thresholds may be exceeded severalfold, the fraction of plasmas hot electrons will be acceptably low ( $<5 \%$ ) with laser wavelengths between 0.26 and $0.53 \mu \mathrm{m}$. A major objective of planned experiments with the Nova laser is to verify that collisional stabilization provides the expected satisfactory plasma conditions for highgain target performance. 


\section{The Nd:Glass Laser}

The Nd:glass laser was chosen in the early 1970 s as the major research tool for laser-plasma interaction research at LLNL. ${ }^{27}$ This choice was based on the system's versatility and scalability and on the potential for harmonic conversion of its fundamental $1.06-\mu \mathrm{m}$ waveleng th to shorter wavelengths. These important features have been used to develop the laser we use today. They include the ease of varying pulse duration and pulse shape, the high optical damage thresholds leading to moderately compact systems, the conveniently available beam diagnostics with film, silicon detectors, or vidicons, and the straightforward size scalability to roughly $100 \mathrm{~kJ}$ through increased beam area or multiple beams. ${ }^{28} \mathrm{~A}$ variety of very efficient harmonic conversion techniques were also invented for this system and are used routinely to produce laser energy for experiments at $0.53,0.35$, and $0.26 \mu \mathrm{m}$. The rapid development of the harmonically converted Nd:glass laser system was motivated by the need for larger-scale experiments and by the poor plasma conditions obtained with $1.06-\mu \mathrm{m}$ and $10.6-\mu \mathrm{m}$ light.

The Nd:glass laser has been : sed for almost all short-wavelength laser-plasma interaction studies so far. However, the krypton fluoride laser, with output at $0.249 \mu \mathrm{m}$, and the harmonically converted atomic-iodine laser, with outputs at 1.3, $0.65,0.44$, and $0.32 \mu \mathrm{m}$, can be used also and are being developed at several laboratories. ${ }^{29,30}$ The $\mathrm{Nd}$ :glass laser in its present embodiment has not been considered for high-repetition-rate $(\sim 5-\mathrm{Hz})$, high-efficiency $(>10 \%)$ applications such as pure- fusion reactor drivers. However, extensions of this technology using crystalline solid state laser media, ${ }^{31}$ as well as gas-laser, ${ }^{32}$ heavy-ion, ${ }^{33}$ and lightion $^{34}$ technologies, are being investigated for this and other applications.

By the early 1970 s, the development of the Nd:glass laser for fusion applications had progressed rapidly from very simple oscillatoramplifier systems to more powerful systems producing a few tens of joules in $0.1 \mathrm{~ns}$. These small, early systems were limited in their output by a nonlinear self-focusing (filamentation) instability of the laser beam. ${ }^{35}$ Subsequent improvements in the underslanding of high-intensity beam propa. gation led to the use of multiple imaging spatial filters $^{36}$ to control self-focusing. As a result longer, larger-diameter amplifier chains were constructed before the beam reached the self-focusing thresholds, and higher output power from each aperture was obtained. Improved understanding of the origin of the nonlinear index of refraction ${ }^{37}$ in uptical materials led to the development of new laser host glasses ${ }^{38}$; improvements in our understanding of optical-surface damage led to new coatings, ${ }^{39}$ which permitted further increases in output power from each aperture. These advances and others led to increases in fluence capability for a given aperture by factors of 5 to 10 over earlier systems. ${ }^{13}$ Examples are the laser chain designs for the Novette (and Nova ${ }^{17}$ ) lasers, which prociuce over $12 \mathrm{TW}$ per beam of focusable power in 100 ps, or over $12 \mathrm{~kJ}$ per beam in $5 \mathrm{~ns}$ at $1.05 \mu \mathrm{m}$.

\section{Harmonic Conversion}

Efficient harmonic conversion to shorter wavelengths is feasible because the phase uniformity of high-power beams from modern lasers is of sufficiently high quality, and the power density is sufficiently high (of the order of gigawatts per $\mathrm{cm}^{2}$ ). In the harmonic generation process, ${ }^{40}$ the incoming light wave drives the nonlinear polarization of the atoms or molecules composing a regular crystai. The magnitude of the linear polarization, given by $P=\chi \varepsilon_{0} E$ (where $\chi$ is the susceptibility, $\varepsilon_{0}$ is the vacuum permittivity, and $E$ is the electric field of the light wave), is $\sim 10^{-4}$ debye/molecule at beam intensities of $1 \mathrm{GW} / \mathrm{cm}^{2}$ (1-MV/cm electric fields). If a non-centrosymmetric crystal is used, the restoring force on the electron cloud is not symmetric about the equiliivrium position, and the susceptibility can become proportional to $E$ :

$\chi=\chi_{0}+\chi_{1} E+\ldots$

Thus there is a term in the polarization, $\chi_{1} \varepsilon_{0} E^{2}$, which leads to an oscillating dipole at twice the fundamental frequency of $E$. A typical $2 \omega_{0}$ nonlinear polarization is $10^{-9}$ debye/molecule. One can imagine this degree of polarization developing in the first plane of atoms in the crystal as the beam strikes the surface.

Since the nonlinear polarization is a factor of $10^{-5}$ less than the linear polarization, and since the 
light intensity is reradiated in all directions from each electron cloud, we must arrange for the radiated waves from each plane of atoms to add coherently in order for the harmonic wave to grow and exceed the intensity of the fundamental driving wave. This is assured by arranging for the phase velocities of the harmonic and fundamental waves to be the same ("phase-matched" by suitably choosing the propagation direction in uniaxial or biaxial crystals. ${ }^{41}$ Phase matching allows for very efficient harmonic conversion when the phase front of the incoming fundamental laser light is uniform enough to maintain these conditions over the entire beam area and through the length of the crystal. For example, with a properly oriented 1.8-cm-thick crystal of potassium dihydrogen phospnate (KDP) we can convert 1.05- $\mu \mathrm{m}$ laser light at $2 \mathrm{GW} / \mathrm{cm}^{2}$ to the second harmonic at $0.53 \mu \mathrm{m}$ with up to $80 \%$ efficiency. (The fundamental frequency is called the first harmonic.) When losses in the optical components (lenses, diagnostics, blast shields, etc.) of a fusion laser beam line are taken into account, it is possible to deliver over
$50 \%$ of the laser's $1.05-\mu \mathrm{m}$ energy to a target at $0.53 \mu \mathrm{m}^{42}$

By using a second crystal after the first crystal, it is also possible to efficiently convert the fundamental $1.05-\mu \mathrm{m}$ radiation to the third or fourth harmonic at $0.35 \mu \mathrm{m}$ or $0.26 \mu \mathrm{m}$, respectively. For the third-harmonic process, the second crystal is oriented to phase-match a one-to-one photon mix of two input waves at 0.53 and $1.05 \mu \mathrm{m}$, which beat together via the $x_{1}$ term in Eq. (2) to produce the output wave at $0.35 \mu \mathrm{m}$. Experiments at Rochester $^{43}$ and at Livermore ${ }^{44}$ have demonstrated third-harmonic conversion efficiencies of up to $80 \%$. For the fourth-harmonic process, ${ }^{16,44}$ the second crystal is oriented to harmonically convert a "fundamental" $0.53-\mu \mathrm{m}$ wave and an output $0.26-\mu \mathrm{m}$ wave. For the $0.26-\mu \mathrm{m}$ experiments mentioned in "Large-Scale Plasmas," above, the maximum energy was restricted to $1.5 \mathrm{~kJ}$ of light in a 1-ns pulse because of low optical-material damage thresholds. This energy was delivered to targets from one of the Novette laser chains.

\section{Inertial Confinement Fusion}

We used the $0.53-\mu \mathrm{m}$ output from Novette to ablatively implode DT fusion fuel to densities of $20 \mathrm{~g} / \mathrm{cm}^{3}$, or 100 times the density of solid DT fuel. ${ }^{45}$ The compression conditions demonstrated in these experiments, together with improved targets and laser pulse shaping, will be used in upcoming experiments on the Nova laser to demonstrate fuel densities approaching 1000 times that of liquid DT $\left(200 \mathrm{~g} / \mathrm{cm}^{3}\right)$. We will also show that these target conditions can be extended to targets designed for use with a future multi-megajoule driver to demonstrate efficient DT burn and energy gain (energy gain is defined as the ratio of the thermonuclear energy produced to the input laser energy). For many applications, such as electricity production, gain of over 100 are needed. Highgain conditions occur when 2 to $10 \mathrm{mg}$ of DT fuel is compressed to a density exceeding $200 \mathrm{~g} / \mathrm{cm}^{3}$ (electron and ion densities exceeding $5 \times 10^{25} \mathrm{~cm}^{-3}$ ) and when the thermonuclear burn of the fuel is initiated in a high-temperature $\left(T_{\text {ion }}=3\right.$ to $\left.5 \mathrm{keV}\right)$, central hot spot. ${ }^{3,46,47} \mathrm{To}$ assemble the fuel in such a state, a precisely time-programmed pressure pulse exceeding $5 \times 10^{7}$ atm (50 Mbar) at peak must be appiied over $10 \mathrm{~ns}$. Very little preheat (increase in entropy) of the fusion fuel can be tolerated if efficient implosions are to occur. In initial experiments, it is expected that 5 to $10 \mathrm{MJ}$ of short-wavelength laser energy $(\lambda<0.5 \mu \mathrm{m})$ will be required to achieve fusion gain approaching 100 . We expect to obtain target gains exceeding 100 with lower input energy as technology and our understanding of the appropriate physics progresses. ${ }^{48}$

There are two laser-driven ICF techniques for generating the required pressures for compression and ignition: "direct drive,"3,49 in which electrons transport the laser energy to the ablation surface, and "indirect drive," in which $x$ rays transport the energy to the ablation surface. Critical issues that are determining the viability of both approaches are symmetry, stability, fuel preheat, and implosion efficiency. ${ }^{27,50)}$ 
In the direct-drive approach, the laser driver energy is focused on and absorbed at the surface of a spherical ablator surrounding the fuel container. Heating and subsequent mass ablation generates the pressure that drives the implosion. The pressure that can be generated depends strongly on the absorbed laser intensity; theory and experiment show that ${ }^{51}$

$P_{a b l} \propto P^{0.5-0.6}$.
In the indirect-drive approach, the principal focus of ICF research at Livermure, the incoming laser light is converted to soft thermal $x$ rays in a highatomi-number hohlraum: the $x$ rays in turn drive the target capsule. This conversion process can be efficient if submicrometer light is used. ${ }^{20}$ An advantage of this approach is that it can produce a highly symmetric implosion with a relativeiy poor laser focal distribution. It also does not require uniformly arrayed laser beams, an advantage for $1 C F$ reactor designs.

\section{Compression}

The laser-plasma coupling achieved on the LLNL Novette laser, operating at $0.53 \mu \mathrm{m}$, represented a significant improvement from that achieved on the earlier LLNL Shiva 1.06- $\mu \mathrm{m}$ laser. We took advantage of this improved coupling to ablatively implode fusion capsules to substantially higher densities than were possible on Shiva for similar capsule designs.

To measure the compression of the imploded target, our colleagues at LLNL ${ }^{45}$ measured the areal density $p \Delta r$ (the product of density and thickness) of the compressed fuel container. The container is referred to as the capsule pL her because of its role in the compression processes. When the target is driven to nigh density, both the pusher $\rho \Delta r$ and the fuel density increase significantly. This is in contrast to strongly preheated targets, such as the so-called exploding-pusher targets first used in ICF demonstrations, ${ }^{34,52}$ in which the pusher $\mathrm{p} \Delta \mathrm{r}$ remains relatively unchanged. In our high-density implosions, $14-\mathrm{MeV}$ neutrons are produced by the fusion of DT near the time of peak compression of the target. Activation of the pusher material by these neutrons ${ }^{53}$ is proportional to the pusher $p \Delta r$ at that time. We infer the fuel density by comparing the measured pusher activation with simple analytical models and with sophisticated hydrodynamic code calculations.

As shown in Fig. 4, we measured increases in the pusher $\rho \Delta r$ over its initial value by factors as great as 60 to 80 when using $0.53-\mu \mathrm{m}$ irradiation. This is 3 to 5 times more pusher compression than in similar $1.06-\mu \mathrm{m}$ experiments on Shiva. Although we do not measure the fuel density directly, these results imply compressed fuel densities of over $20 \mathrm{~g} / \mathrm{cm}^{3}$. By using 30 to $50 \mathrm{~kJ}$ of $0.35-\mu \mathrm{m}$ light from the Nova laser to extend these results, we are confident that we will achieve the DT fuel densities required for high-gain target operation.

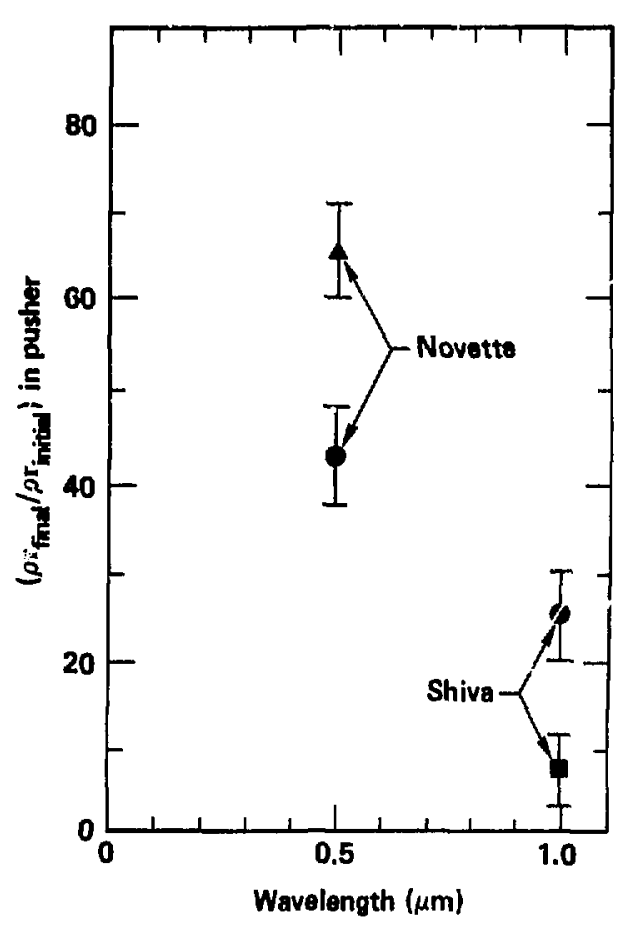

Fig. 4. A direct measurement of the areal density of the DT fuel container (pusher) using neutronactivation technicues shows a 3-fold increase over earlier results. From this increase we infer that the compression is ablative and that the DT fuel reached $>100$ times liquid-DT density. 


\section{X-Ray Lasers}

We have recently observed ${ }^{54,55}$ stimulated emission in the soft $x$-ray region. The experiments utilized 2-TW, 450-ps pulses of $0.53-\mu \mathrm{m}$ light from the Novette laser to irradiate thin-foil selenium targets up to $2.2 \mathrm{~cm}$ long at a power density of $5 \times 10^{13} \mathrm{~W} / \mathrm{cm}^{2}$. The ability to irradiate candidate $x$-ray laser targets over longer dimensions $(>1 \mathrm{~cm})$ and to couple the laser light to higherdensity plasmas than in previous experiments was crucial in obtaining sufficient single-pass gain to make this unambiguous observation. Other important advances leading to these observations were in the areas of $x$-ray diagnostics, target fabrication, and modeling.

The prospect of generating laser action at $x$-ray wavelengths has been discussed since the invention of the laser. ${ }^{56}$ Although many potential schemes have been identified that could lead to gain at wavelengths near $100 \AA$ (see references in Refs. 54 and 55), many difficulties have prevented unambiguous confirmation. One of the most demanding requirements has been to find a plasma mediun with a sutficient gain-length product to produce measurable line enhancement on a single pass: there are at present no suitable cavity configurations to provide multiple passes through the gain medium. ${ }^{57}$ Other physical constraints arise because the wavelength scaling of the small-signal gain (for fixed Einstein $A$ coefficient) is proportional to $\lambda^{3} T_{j}^{-1 / 2}$, because suitable soft $x$-ray transitions have short lifetimes $\left(<5 \times 10^{-11} \mathrm{~s}\right)$, and because the efficiency of channeling pump energy into the upper laser state is inherently low. ${ }^{58}$ These considerations lead to the requirement that the pump power exceed $10^{13} \mathrm{~W} / \mathrm{cm}^{2}$. For net laser gain, it is also necessary thit the propagation path be uniform enough to prevent refraction of the beam out of the gain medium and that there be no absorption losses from other ionic transitions. Finally, time-resolved and spectrally resolved diagnostics are required to record gain in the presence of intense background emission from neighboring transitions that occur during the laser pulse and in the afterglow of the relaxing plasma. ${ }^{59}$

The tachnique used in these experiments for producing $x$-ray gain is the electron collisional $e x-$ citation of neon-like ions. It has long been recog- nized that the large difference in the radiative decay rates of the $2 p^{5} 3 p$ and $2 p^{5} 3 s$ states in such ions can lead to population inversion between these states in an optically thin plasma. ${ }^{60}$ Calculations based on isoelectronic scaling of the neon-like configuration in higher- $Z$ ions showed that, with a sufficiently high pumping rate, gain at XUV and soft $x$-ray wavelengths was possible. ${ }^{58}$ The required pumping rates at sufficient electrun density and over a sufficient length of active ions were achieved by bringing the two opposing Novette laser beams to a common line focus with a combination of cylindrical and spherical lenses.

Uniform neon-like plasmas with the proper density conditions were generated using so-called exploding-foil targets. These targets, originally designed for the plasma pirysics studies described earlier, are composed of a thin foil of selenium (750 $\AA$ ) on a thin Formvar backing (i500 $\AA$ ) with a density-thickness product of about $4 \times 10^{-5} \mathrm{~g} / \mathrm{cm}^{3}$. They explode because of the rapid heating and ablation of the entire target volume. Using a holographic interferometer with 20-ps time resolution, scientists at KMS Fusion confirmed the expected density profile of the expanding selenium plasmas. They demonstrated a transverse density scalelength $[(1 / n)(d n / d x)]^{-1}$ of $100 \mu \mathrm{m}$ at $n_{\mathrm{e}}=3 \times$ $10^{20} \mathrm{~cm}^{-3}$; this was sufficiently uniform to prevent refraction of $200-\AA$ photons out of the laser channel, which was $1 \mathrm{~cm}$ long and about $100 \mu \mathrm{m}$ wide.

High amplification of the neon-like $2 \mathrm{p}^{5} 3 \mathrm{p}$ $2 p^{5} 3$ s transitions in selenium can be readily seen in Fig. 5(a), which shows the x-ray spectrum along the plasma axis under maximum-gain conditions. The lines at 206 and $209 \AA$ dominate the spectrum. The spectrum in Fig. 5(b) was recorded on the same shot as Fig. 5(a) with an instrument looking transverse to the plasma axis, but recording the total radiating plasma volume. The lines at 206 and $209 \AA$ were not observed in this short transverse direction, because they were not substantially amplified.

The time-resolved measurements shown in Fig. 6 were made with an absolutely calibrated spectrometer. They indicate a lower bound of 35 to $50 \mathrm{keV}$ for the brightness temperature of these lines. This brightness temperature is consistent 


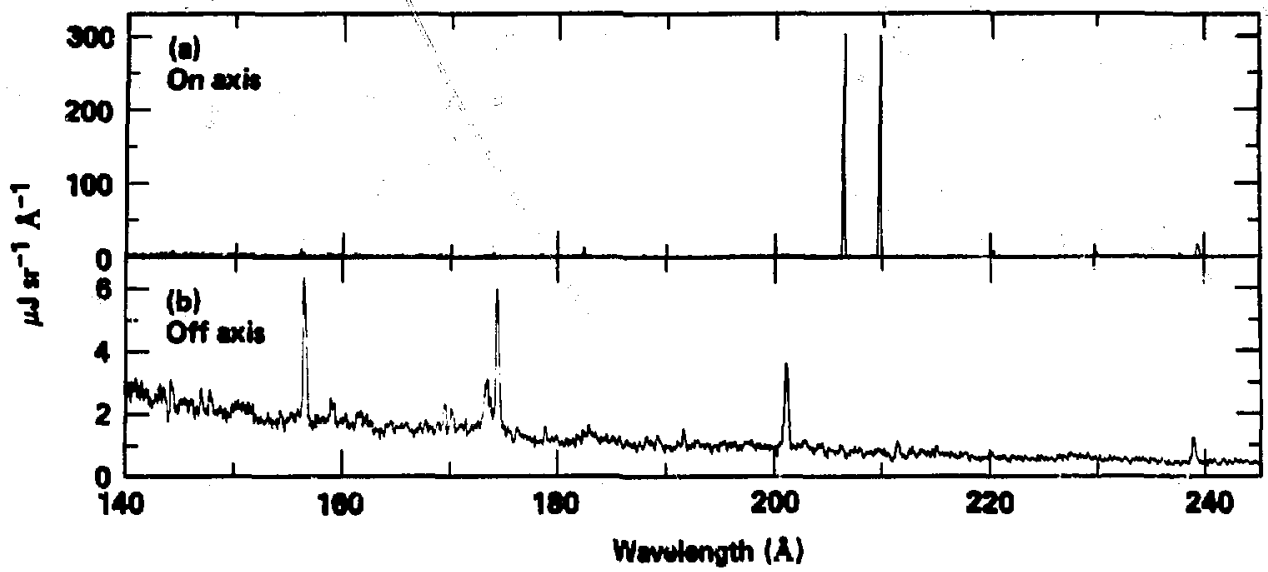

Fig. 5. On-and off-axis soft $x$-ray spectra from a 2.2-cm-long selenium laser, obtained with a time-gated grazing-incidence spectrograph (data accumulated from the beginning of radiative output until $\sim 500$ ps after the peak of the pumping laver pulse). (a) On-axis spectrum shows the two strongest lines at maximum gain. (b) Off-axis spectrum shows the output from the entire plasm, viewed at an angle $73^{\circ}$ to the laser gain axis; laser lines are too weak and spontanewus to be visible. (The absolute intensity scale for the off-axis data is approximate.)

(a)

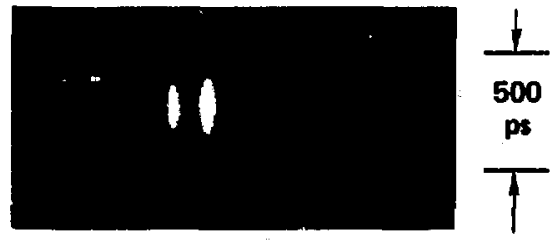

(b)

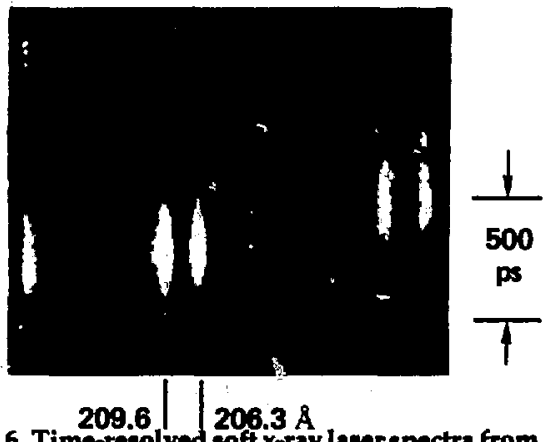

Fig. 6. Time-resolved soft $x$-ray laver spectra from selenium lasers (a) $2.2 \mathrm{~cm}$ long, (b) $1.1 \mathrm{~cm}$ long. In (b), higher sensitivity makes lines from other transitions visible. with $x$-ray gain, and is not consistent with thermal processes associated with the plasma electron temperature of less than $1 \mathrm{keV}$ or with the intensity of nearby lines, which are not amplified but have equivalent temperatures of about $0.1 \mathrm{keV}$. A small-signal gain coefficient of about $6 \mathrm{~cm}^{-1}$ was inferred for both lines by means of experiments in which the plasma length was varied from 0.1 to $1.2 \mathrm{~cm}$. Similar experiments with 29-times ionized yttrium produced equivalent brightness temperatures of about $10 \mathrm{keV}$ at $155 \AA$, demonstrating isoelectronic scaling of the neon-like transitions to shorter wavelengths.

The peak-output spectral brightness from this laser system is $2 \times 10^{11} \mathrm{~W} \cdot \mathrm{cm}^{-2} \AA^{-1} \mathrm{sr}^{-1}$, which was obtained from a $2.2-\mathrm{cm}$-long plasma irradiated by laterally displacing two opposed $1.1-\mathrm{cm} \times 0.01-\mathrm{cm}$ focused Novette beams to excite a $2.2-\mathrm{cm}$-long active region. The peak brightnesses from these small x-ray sources are $10^{10}$ to $10^{11}$ times greater than today's most powerful $x$-ray generators and synchrotron sources. ${ }^{61}$ However, they are presently one-st. '. short-pulse devices that need a great deal more effort before they become useful laboratory $x$-ray sources. 


\section{Summary}

Experiments using the harmonically converted Novette $N d$ :glass laser at 0.53 and $0.26 \mu \mathrm{m}$ demonstrated satisfactory coupling of laser light to 0.1 to $1.0-\mathrm{cm}$-sized fusion and $\mathrm{x}$-ray laser targets. The coupling of short-wavelength laser light to these plasmas is well understood and is primarily collisional, in contrast to previous experiments at $1.06 \mu \mathrm{m}$ and $10 \mu \mathrm{m}$, in which the coupling was collective. The coupling improvements led directly to the demonstration of higher-density, ablative implosions of DT fusion fuel and to the first unambiguous observation of amplified stimulated emission in the sott $x$-ray region. The Nova laser (Fig. 7), providing 30- to 50-TW output at 0.53 and
$0.35 \mu \mathrm{m}$, will be used to extend laser-plasma studies to plasmas several times larger than those used on Novette. We expect that these experiments will confirm our theoretical and experimental understanding of the laser-plasma coupling and of the implosion physics of the capsules that will be required for high-gain operation. We also expect to extend $x$-ray laser performance to shorter wavelengths, higher power, and increased spatial coherence, and to conduct a variety of shock-wave, atomic-physics, and hydrodynamic experiments that became possible with the development of multi-terawatt, short-wavelength lasers. 
(a)

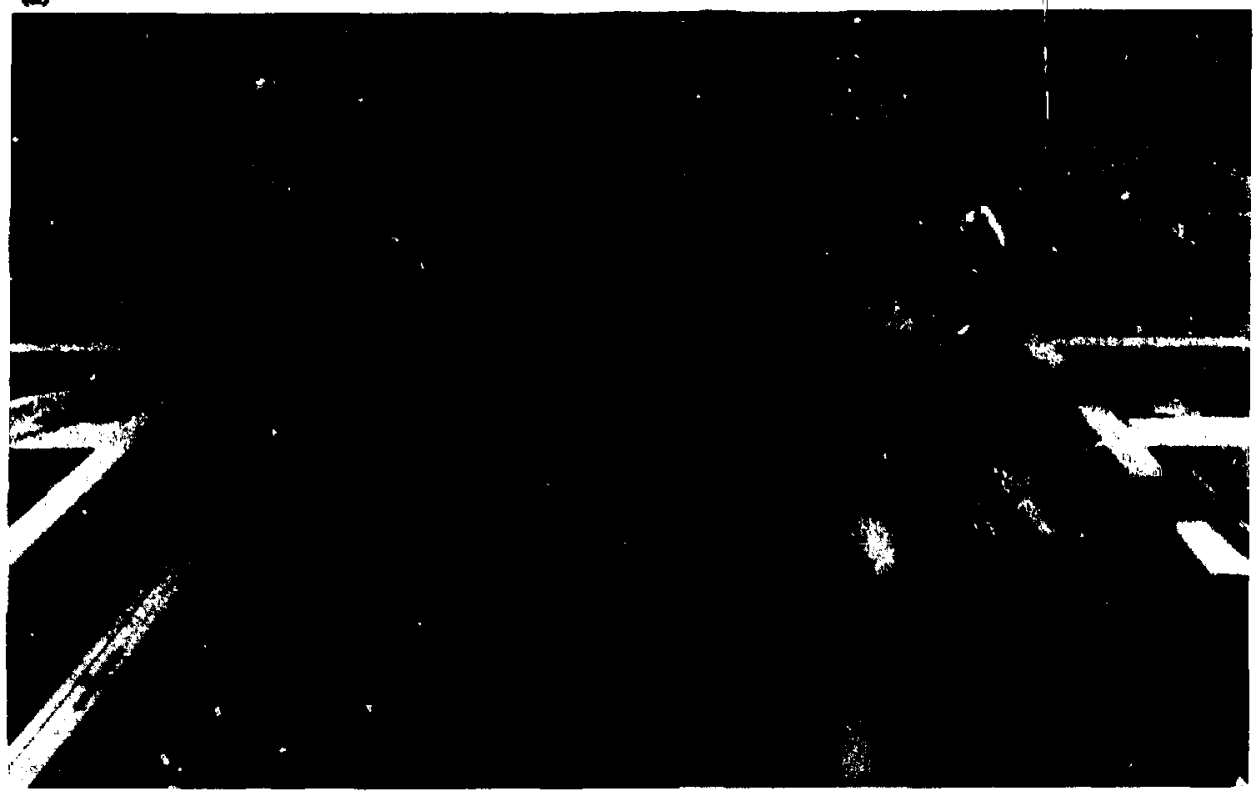

(b)

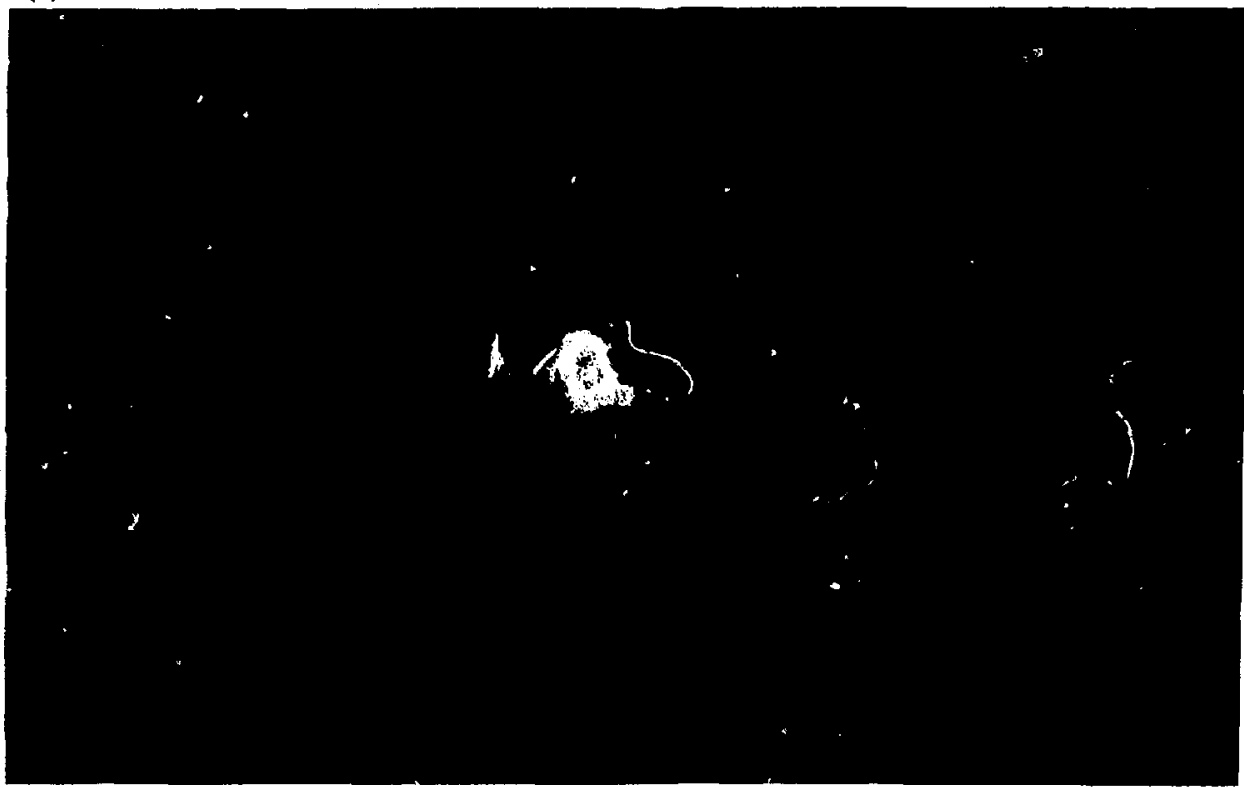

Fig. 7. (a) The ten-beani Nova lace is mounted with five beams on each spaceframe. Nova will develop $>100 \mathrm{~kJ}$ at $1.05-\mu \mathrm{m}$ and 50 to $70 \mathrm{~kJ}$ at 0.53 and $0.35 \mathrm{~mm}$. (b) Nova's $5.3-\mathrm{m}$-diam target chamber is being readied for experiments that are to start in the spring of 1985 . Here, 5 of the 10 harmonic-conversion and focusing modules are seen mounted on the target chamber. 


\section{References}

1. S. Colgate, R. E. Kidder, J. H. Nuckolls, R. F. Zabawski, ard E. Teller, Lawrence Livermore National Laboratory, Livermore, CA unpublished calculations (1961).

2. N. G. Basov and O. N. Krokhin, in Proc. 3rd Quantum Electron. Int. Conf., Paris, Vol. 2, pp. 1373 (1963), N. Bloembergen and P. Grivet, Eds. (Columbia Univ. Press, New York, NY, 1964).

3. J. H. Nuckolls, L. L. Wood, A. R. Thiessen, and G. B. Zimmerman, Nature 239, 139 (1972).

4. L. I. Gudzenko and L. A. Shelepin, Sov. Phys. JETP 18, 998 (1964).

5. R. W. Waynant and R. C. Elton, Proc. IEEE 64, 1059 (1976).

6. Laser Interaction and Related Plasma Phenomena, H. J. Schwarz and H. Hora, Eds. (Plenum Press, New York, NY, 1971-84), Vols, 1-6.

7. J. W. Shearer et al., Phys. Rev. A6, 764 (1972).

8. G. Charatis et al., in Proc. 5th 1AEA Plasma Fusion Conf., Tokyo, Japan 1974 (Plasma Physics and Controlled Nuclear Fusion Research 19;4, 2, 317 IAEA, Vienna, 1975).

9. K. R. Manes, H. G. Ahlstrom, R. A. Haas, and J. F. Holzrichter, J. Opt. Soc, Am, 67, 717 (1977).

10. For detailed descriptions of laser-plasma investigations, see the Annual Reports from the following laboratories: CEA Limeili; France; KMS Fusion, Inc., Ann Arbor, MI; Naval Research Laboratory, Washington, DC; Osaka Lniversity, Osaka, Japan; Sandia Laboratories, Albuquerque, NM; Lebedev Institute, Moscow, USGR; Max Planck Institute for Plasma Physics, Garching, W. Germany; Rutherford Laboratory, Didcot, England; Lawrence Livermore National Laboratory, Livermore, CA; Los Alamos Scientific Laboratory, Los Alamos, NM; University of Rochester, Laboratory for Laser Energetics, Rochester, NY.

11. C. S. Liu, M. N. Rosenbluth, and R. B. White, Phys. Fluids 17, 1211 (1974).

12. P. K. Kaw, W. L. Kruer, C. S. Liu, and K. Nishikawa, in Advances in Plasma Physics, Vol. 6, A. Simon and W. Thompson, Eds. (Wiley, New York, NY, 1976).

13. D. R. Speck et al., IEEE J. Quantum Electron;. QE-17, 1599 (1981).

14. T. F. Stratton, in High Power Gas Lasers, E. R. Pike, Ed. (The Institute of Physics, London, 1975), 284-311.

15. 1980 Laser Program Annual Report, Lawrence Livermore National Laboratory, Livermore, CA, UCRL50021-80 (1981), Vol. 2, p. 7-1.

16. K. Manes et al., Lawrence Livermore National Laboratory, Livermore, CA, UCRL-90054-Rev. 1 (1984).

17. W. W. Simmons et al., "Nova," in Proc. of the Ninth Symp. on Eng .Problems of Fusion Res., Chicago, IL, Vol. II, pp. 1221-1273, October 1981, (IEEE Pub. No. 81CH1715-2 NPS).

18. S. P. Obenschain et al., Phys. Rev, Lett. 50 (1), 44 (1983).

19. B. Yaakobi et al., Opt. Commun. 39, 175 (1981); and E. Cottet, J. P. Romain, R. Fabbro, and B. Faral, Phys. Rev. Lett. 52, 1884 (1984).

20. R. L. Kauffman et al., Bull. Am. Phys. Soc. 29, 1183 (1984).

21. L. L. Endelman, Ed., Proc. 15th Int. Congr. on High Speed Photog. Photonics (SPIE Vol. 348, 1982).

22. C. E. Max, Physics of Laser Fusion, Vol. I, "Theory of the Coronal Plasma in Laser Fusion Targets," Lawrence Livermore National Laboratory, Livermore, CA, UCRL-53107 (1981).

23. D. W. Forslund, J. M. Kindel, and E. L. Lindman, Phys. Fluids 18, 1002 (1975); D. W. Phillion, E. M. Campbell, K. G. Estabrook, G. E. Phillips, and F. Ze, Phys. Rev. Lett, 49, 1405 (1982); K. Estabrook, W. L. Kruer, and B. F. Lasinski, Phys. Rev. Lett. 45, 1399 (1980), and references therein.

24. C. Joshi et al., Naiure 311, 525 (1984).

25. Discussed in C. E. Max et al., see Ref. 6, Vol. 6, p. 507.

26. R. E. Turner et al., Lawrence Livermore National Laboratory, Livermore, CA, UCRL-91425 (1984); submitted to Phys. Rev. Lett.

27. 1973 to 1984 Laser Program Annual Reports, Lawrence Livermore National Laboratory, Livermore, CA, UCRL-50021-73 to 50021-84, available from the National Technical Information Service, Springfield, VA 22161.

28. J. F. Holzrichter et al., J. Fusion Energy 2 (1), 5 (1982): also available as Lawrence Livermore National Laboratory, Livermore, CA, UCRL-52868, Rev. 1 (1982).

29. E. T. Salesky and W. D. Kimura, Los Alamos National Laboratory, Los Alamos, NM, LA-UR-84-4040 (1984), LA-UR-84-4038 (1984), and LA-UR-84-4039 (1984).

30. G. Brederlow, K. J. Witte, E. Fill, K. Hohla, and R. Volk, IEEE J. Quantum Electron. QE-12, 152 (1976). Also see articles in Ref. 6. 
31. J. L. Emmett, W. F. Krupke, and J. B. Trenhalme, The Future Development of High-Power Solid State Laser Systems, Lawrence Livermore National Laboratory, Livermore, CA, UCRL-53344 (1962) (Vol. IV in Physics of Laser Fusion):

32. C. K. Rhedes, Ed., Excimer Lasers (Springer-lerlag, New York, NY, 1984), 2nd ed. (vol. 30 in Topics in Applied Physics).

33. "Heavy Ion Accelerators and Their Applications to Inertial Fusion," conf. proc. (Institute for Nuclear Study, Tokyo, 1984).

34. I. Pace Van Devender et al., to be published in Laser and Part. Beams, (April 1985).

35. V. I. Bespalov and V. I. Talanov, JETP Lett. 3, 307 (1966)

36. W. W. Simmons et al, , IEEE J. Quantum Electron. QE-11, 31D (1975); A. Bettinger, C. Charles, J. Osmalin, and J. G. Giraud, Opt. Commun. 18, 176(1976); and J. T. Hunt, J. A. Glaze, W. W. Simmons, and P. A. Renard, Appl. Opt. 17, 2053 (1978).

37. N. L. Boling, A. J. Glass, and A. Owyoung, IEEE J. Quantum Electron. QE-14, 601 (1978).

38. S. E. Stokowski, R. A. Saroyan, and M. J. Weber, Nd-Doped Laser Glass: Spectroscopic and Physical Proper. lies, Lawrence Livermore National Laboratory, Livermore, CA, Special Publication M-95, Rev. 2 (1981).

39. Proc. Symp. on "Laser Induced Damage in Optical Materials" National Bureau of Standards Special Publications, 1976 to 1984.

40. P. A. Franken, A. E. Hill, C. W. Peters, and G. Weinreich, Phys. Rev. Lett. 7, 118 (1961).

41. J. A. Giordmaine, Phys. Rev. Lett. 8, 19 (1962).

42. M. A. Summers et al., Lawrence Livermore National Laboratory, Livermore, CA, Conf-811040 (1982), pp. 78-88; D. Eimerl, in Energy Technol. Rev., Lawrence Livermore National Laboratory, Livermore, CA, UCRL-52000-82-8 (August 1982); D. Eimerl, Lawrence Livermore National Laboratory, Livermore, CA, UCRL-92087, to be published in Proc. Lasers '84 (Society of Optical and Quantum Electronics).

43. W. Seka et al., Opt. Commun. 34, 469 (1980); R. S. Craxton, Opt. Commun. 34, 474 (1980).

44. G. J. Linford et al., Appl. Opt. 21, 3633 (1982) and Appl. Opt. 22, 1957 (1983).

45. F. Ze et al., Lawrence Livermore National Laboratory, Livermore, CA, UCRL-91087 (1984); submitted to Nucl. Fusion.

46. J. L. Emmett, J. H. Nuckolls, and L. L. Wood, Sci. Am. 23n, 24 (June 1974).

47. J. Meyer-Ter-Kehn, Nucl. Fusion 22, 561 (1982).

48. J. H. Nuckolls, Phys. Today 35, 24 (Sept. 1982).

49. S. E. Bodner, J. Fusion Energy 1, 221 (1981).

50. C. E. Max, J. D. Linal, and W. C. Mead, Nucl. Fusion 23, 131 (1983).

51. T. J. Goldsack et al., Opt. Commun. 42, 55 (1982).

52. E. K. Storm et al., Lawrence Livermore National Laboratory, Livermore, C.A, UCRL-50021-76 (1977), p. 5-52, and UCRL $50021-77$ (1978), vol. 2, p. 6-38.

53. E. M. Campbell et al., J. Appl. Phys. 51, 6062 and 6065 (1980).

54. D. L. Matthews et al., Phys. Rev. Lett. 54, 110 (1985).

55. M. D. Rosen et al., Phys. Rev. Lett. 54, 106 (1985).

56. A. L. Schawlow and C. H. Townes, Phys. Rev. 112, 1940 (1958).

57. In recent work, Barbee has made $x$-ray mirrors with greater than $50 \%$ reflectivity at normal incidence near $200 \dot{\mathrm{A}}$. Laser cavities may be made with these devices.

58. P. L. Hagelstein, Plasma Phys. 25, 1345 (1983).

59. N. M. Ceglio, "Measurements of Amplified Spontaneous Emission at $200 \dot{A}$," in Proc. 5th Topical Conf. on High Temp. Plasma Diagn. (2984), to be published in Rev. Sci. Instrum.

60. A. V. Vinogradov, I. Sobelman, and E. Yukov, Sov, J. Quantum Electron. 7, 32 (1977); P. L. Hagelstein, Ph.D. Thesis, Massachusetts Institute of Technology, Cambridge, MA: Lawrence Livermore National Laboratory, Livermore, CA, UCRL-53100, (1981); A. V. Vinogradov and V. Shlyaptsev, Sov. J. Quantum Electron. 13, 1511 (1983), and references therein.

61. C. K. Rhodes, Proc, on Vacuum UV and X-ray Sources, Proc. of the Nat. Acad. Sci. (Nov. 9, 1984). 